\title{
Erratum: Paraxial skyrmionic beams [Phys. Rev. A 102, 053513 (2020)]
}

\author{
Sijia Gao, Fiona C. Speirits, Francesco Castellucci, Sonja Franke-Arnold, Stephen M. Barnett, and Jörg B. Götte 무
}

(Q) (Received 21 September 2021; published 8 October 2021)

DOI: 10.1103/PhysRevA.104.049901

The effective magnetization in Fig. 2(c2) is wrongly plotted in the original paper. The correct pattern is shown in the corrected figure in this Erratum.

The explicit expressions for the three components of the effective magnetization are as follows:

$$
\begin{aligned}
& M_{x}=\frac{2 \sqrt{\frac{\left|\ell_{0}\right| !}{\left|\ell_{1}\right| !}}\left(\frac{\rho \sqrt{2}}{w(z)}\right)^{\left|\ell_{1}\right|-\left|\ell_{0}\right|}}{1+\frac{\left|\ell_{0}\right| !}{\left|\ell_{1}\right| !}\left(\frac{2 \rho^{2}}{w(z)^{2}}\right)^{\left|\ell_{1}\right|-\left|\ell_{0}\right|}} \cos \left[\left(\ell_{1}-\ell_{0}\right) \phi-\left(\left|\ell_{1}\right|-\left|\ell_{0}\right|\right) \xi(z)\right] \\
& M_{y}=\frac{\left.2 \sqrt{\frac{\left|\ell_{0}\right| !}{\left|\ell_{1}\right| !}} \frac{\rho \sqrt{2}}{w(z)}\right)^{\left|\ell_{1}\right|-\left|\ell_{0}\right|}}{1+\frac{\left|\ell_{0}\right| !}{\left|\ell_{1}\right| !}\left(\frac{2 \rho^{2}}{w(z)^{2}}\right)^{\left|\ell_{1}\right|-\left|\ell_{0}\right|}} \sin \left[\left(\ell_{1}-\ell_{0}\right) \phi-\left(\left|\ell_{1}\right|-\left|\ell_{0}\right|\right) \xi(z)\right] \\
& M_{z}=-1+\frac{2}{1+\frac{\left|\ell_{0}\right| !}{\left|\ell_{1}\right| !}\left(\frac{2 \rho^{2}}{w(z)^{2}}\right)^{\left|\ell_{1}\right|-\left|\ell_{0}\right|}} .
\end{aligned}
$$

For the particular example of the $\ell_{0}=0$ and $\ell_{1}=1$ case, these components simplify to

$$
\begin{aligned}
& M_{x}=\frac{2 \frac{\rho \sqrt{2}}{w(z)}}{1+\frac{2 \rho^{2}}{w(z)^{2}}} \cos [\phi+\xi(z)] \\
& M_{y}=\frac{2 \frac{\rho \sqrt{2}}{w(z)}}{1+\frac{2 \rho^{2}}{w(z)^{2}}} \sin [\phi+\xi(z)] ; \\
& M_{z}=-1+\frac{2}{1+\frac{2 \rho^{2}}{w(z)^{2}}} .
\end{aligned}
$$

With these components the polarization and effective magnetization in Fig. 2(c2) agree.

We would also like to clarify the units of the propagation distance $z$ in Figs. 2 and 5 of the original article. The distances $z= \pm 10$ correspond to half a Rayleigh length $z_{R}$ on either side of the the focus at $z=0$. Correspondingly, $z= \pm 2$ in Fig. 5 corresponds to $\pm z_{R} / 10$.

We are grateful to Dr. Yijie Shen for alerting us to this error. 


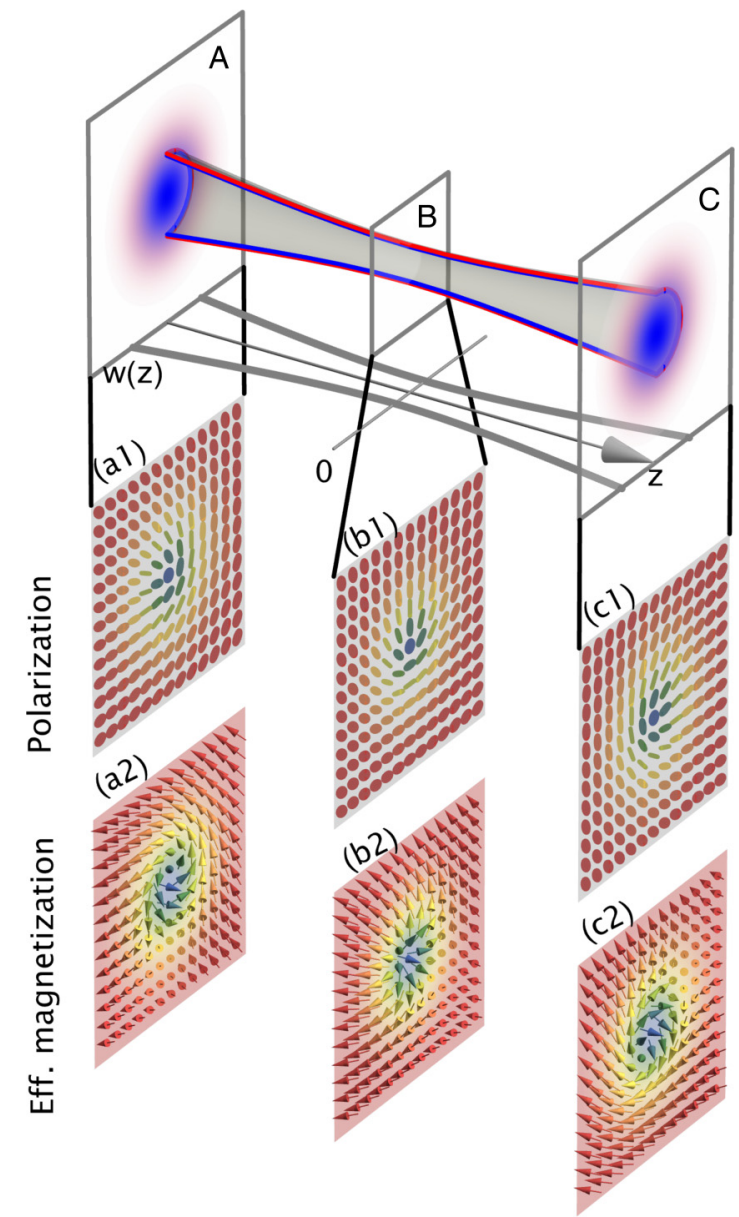

FIG. 2. Polarization structure for a superposition of LG modes with $\ell_{1}=1$ and $\ell_{0}=0$ focused at $z=0$. The beam surface separating the regions in which the modes have the larger amplitude, $u_{0}$ (blue) and $u_{1}$ (red). $\mathrm{A}, \mathrm{B}$, and $\mathrm{C}$ are three cross sections of interest, at $z=-z_{R} / 2,0$ and $z_{R} / 2$, respectively. (a1), (b1), and (c1) are spatially varying polarization patterns corresponding to each plane, while (a2), (b2), and (c2) are the corresponding effective magnetizations, with the classic chiral and hedgehog forms, respectively. 